\title{
Alta frecuencia de hipovitaminosis D y baja densidad mineral ósea en un grupo de hombres con infección por VIH en Chile
}

\author{
Carlos Pérez y María Elena Ceballos
}

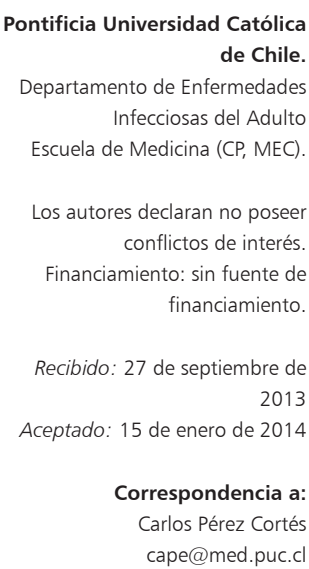

Pontificia Universidad Católica de Chile. Departamento de Enfermedades Infecciosas del Adulto Escuela de Medicina (CP, MEC).

Los autores declaran no poseer conflictos de interés. Financiamiento: sin fuente de

financiamiento.

Recibido: 27 de septiembre de

Aceptado: 15 de enero de 2014

Correspondencia a: Carlos Pérez Cortés cape@med.puc.cl

\section{High frequency of hypovitaminosis D and low bone mineral density in a sample of HIV-infected men in Chile}

Objectives: The aim of this study was to determine the frequency of hypovitaminosis $\mathrm{D}$ and low bone mineral density in a sample of patients within our HIV cohort. Methods: A random sample of 16 HIV-infected patients was selected for evaluation of levels of Vitamin $\mathrm{D}$ and bone mineral density with dual X ray absorptiometry. $R e-$ sults: Within this sample of patients, 94\% had low levels of vitamin D. Among them, eight had insufficient levels (10-30 ng/mL) and seven had deficient levels (less than $10 \mathrm{ng} / \mathrm{mL})$ of this vitamin. Ten out of sixteen $(63 \%) \mathrm{had}$ an abnormal dual X ray absorptiometry (osteopenia or osteoporosis). All patients with osteopenia or osteoporosis had low levels of vitamin D, were on CDC stage A, had a CD4+ count greater than $350 \mathrm{cells} / \mathrm{mm}^{3}$ and HIV viral load less than 50 copies/mL. Conclusions: Low bone mineral density and hypovitaminosis D is highly frequent in this sample of HIV-infected patients in Chile.

Key words: Bone density, HIV, osteoporosis, osteopenia, vitamin D.

Palabras clave: Densidad mineral ósea, VIH, osteoporosis, osteopenia, vitamina D.

\section{Introducción}

$\mathrm{D}$ istintos estudios han mostrado una alta frecuencia de hipovitaminosis $\mathrm{D}$ en individuos con infección por virus de inmunodeficiencia humana (VIH), particularmente en pacientes que reciben efavirenz $\mathrm{e}$ inhibidores de proteasa ${ }^{1,2}$.

Junto con ello, existe una alta prevalencia de baja densidad mineral ósea en estos pacientes. Se ha reportado seis veces más riesgo de osteopenia y 3,7 veces más riesgo de osteoporosis comparado con la población general ${ }^{3}$. También son más frecuentes las fracturas óseas en pacientes con infección por VIH comparado con individuos sanos de la misma edad ${ }^{4,5}$.

Algunos factores que se han asociado independientemente con pérdida de densidad mineral en esta población han sido el virus en sí mismo, la edad, tabaquismo, abuso de alcohol, bajo índice de masa corporal y particularmente, el uso de terapia antirretroviral (TARV) ${ }^{6-9}$. En Latinoamérica no se han publicado datos sobre este tema en pacientes con VIH.

El objetivo de este estudio fue determinar la frecuencia de hipovitaminosis D y de densidad mineral ósea disminuida en una muestra de pacientes con infección por VIH en la cohorte de pacientes que se atienden en la Red de Salud de la Pontificia Universidad Católica de Chile.

\section{Métodos}

En la Red de Salud de la Pontificia Universidad Católica seguimos una cohorte de más de 1.000 pacientes con infección por VIH que viven principalmente en Santiago, Chile. Una muestra aleatoria de 16 pacientes fue seleccionada para evaluar la densidad mineral ósea y los niveles de vitamina D como parte de su control rutinario de salud.

Se registraron datos demográficos, comorbilidades, uso de drogas ilícitas, hábitos, historia de fracturas óseas, estado VIH/SIDA del CDC, media de linfocitos CD4 y carga viral de VIH.

Se definió el realizar ejercicio físico en forma regular como hacer ejercicio tres veces a la semana; el tabaquismo activo como consumo de al menos un cigarrillo al día y la ingesta de alcohol ocasional a quien refería ingerir alcohol uno o dos días a la semana.

Se solicitaron exámenes generales como creatinina sérica $(\mathrm{mg} / \mathrm{dl})$, fosfatasas alcalinas $(\mathrm{U} / \mathrm{l})$, calcio $(\mathrm{mg} / \mathrm{dl})$, fósforo ( $\mathrm{mg} / \mathrm{dl}$ ), niveles de 25-hidroxi-vitamina D (25OHD) $(\mu \mathrm{g} / \mathrm{ml})$ por medio de un inmunoensayo competitivo quimioluminiscente (CLIA) en una plataforma automatizada (Liaison ${ }^{\circledR}$ Diasorin). Se evaluó la densidad mineral ósea a través de densitometría (DXA). Ochenta y siete por ciento de los pacientes fueron estudiados durante el invierno.

Se definió como insuficientes los niveles de vitamina 
D entre 10 y $30 \mu \mathrm{g} / \mathrm{ml}$ y deficientes a valores menores a $10 \mu \mathrm{g} / \mathrm{ml}^{10}$.

Los parámetros demográficos fueron analizados por estadísticas descriptivas. En variables categóricas se usaron porcentajes y en variables continuas se utilizaron medias y promedios.

\section{Resultados}

Los 16 pacientes eran de sexo masculino. La media de edad fue de 46 años, con un rango entre 29 y 60 años. El 50\% hacía ejercicio en forma regular, 44\% fumaban, $78 \%$ tomaba alcohol en forma ocasional y ninguno usaba drogas ilícitas.

Todos los pacientes estaban recibiendo TARV y 10 de ellos ingerían otros medicamentos (principalmente estatinas, metformina o inhibidores selectivos de la recaptación de serotonina). El 63\% de los pacientes presentaba comorbilidades, mayormente dislipidemia y depresión. Un paciente tenía co-infección con el virus de la hepatitis B. No hubo pacientes con historia de fracturas óseas. El tiempo desde el diagnóstico hasta el inicio de la TARV fue de 1,9 años en promedio. Un $81 \%$ de los pacientes se encontraba en etapa A del CDC al momento de iniciar la TARV. Al momento del estudio, la media de linfocitos CD4 fue de 532 céls $/ \mathrm{mm}^{3}$ y 14 de 16 pacientes $(88 \%)$ tenían carga viral VIH menor a 50 copias $/ \mathrm{ml}$. Todos los pacientes presentaban función renal normal y niveles adecuados de calcio sérico. Tres pacientes tenían niveles bajos de fósforo y tres niveles elevados de fosfatasas alcalinas. Quince de 16 pacientes (94\%) presentaron niveles bajos de 25OHD. Entre ellos, 8 tenían niveles insuficientes $(10-30 \mu \mathrm{g} / \mathrm{ml})$ y 7 tenían niveles deficientes (menor a 10 $\mu \mathrm{g} / \mathrm{ml})$. Diez de los 16 pacientes (63\%) mostraron una densitometría ósea anormal (3 con osteoporosis lumbar y el resto con osteopenia de la columna lumbar y/o caderas) (Tabla 1).

El subgrupo de los 10 pacientes que presentó osteopenia u osteoporosis tenían entre 29 y 60 años (cuatro de ellos tenían 50 años o más), 70\% era sedentario, 50\% fumadores y $90 \%$ tomaba alcohol en forma ocasional. Ninguno de ellos presentaba una excesiva ingesta de

Tabla 1. Características clínicas y de laboratorio de pacientes con infección por VIH estudiados con niveles de vitamina D y densidad mineral ósea. Pontificia Universidad Católica de Chile

\begin{tabular}{|c|c|c|c|c|c|c|c|}
\hline $\begin{array}{l}\text { Paciente } \\
\text { n }\end{array}$ & $\begin{array}{l}\text { Edad } \\
\text { (años) }\end{array}$ & $\begin{array}{l}\text { Etapa } \\
\text { CDC }\end{array}$ & Historia TARV & $\begin{array}{l}\text { Creatinina } \\
\text { sérica }(\mathrm{mg} / \mathrm{dl})\end{array}$ & $\begin{array}{l}\text { Calcio/fósforo } \\
\text { sérico }(\mathrm{mg} / \mathrm{dl})\end{array}$ & $\begin{array}{l}\text { Niveles de } \\
25 \text { OHD }(\mu \mathrm{g} / \mathrm{ml})\end{array}$ & Densitometría ósea \\
\hline 1 & 30 & $\mathrm{~A} 2$ & zidovudina/lamivudina/efavirenz & 0,6 & $9,4 / 3,0$ & 10,5 & Osteopenia cadera izquierda \\
\hline 2 & 47 & A3 & zidovudina/lamivudina/efavirenz & 0,79 & $9,5 / 2,6$ & 8,6 & Osteoporosis lumbar \\
\hline 3 & 45 & $\mathrm{C} 3$ & $\begin{array}{l}\text { lamivudina/indinavir/ritonavir } \rightarrow \text { tenofovir/lamivudina/ } \\
\text { darunavir/ritonavir/efavirenz }\end{array}$ & 0,99 & $9,1 / 3,4$ & 33,7 & Normal \\
\hline 4 & 50 & $\mathrm{C} 3$ & $\begin{array}{l}\text { zidovudina/lamivudina/nelfinavir } \rightarrow \text { zidovudina/ } \\
\text { lamivudina/atazanavir }\end{array}$ & 0,79 & $9,5 / 3,3$ & 16 & $\begin{array}{l}\text { Osteoporosis lumbar y } \\
\text { osteopenia caderas }\end{array}$ \\
\hline 5 & 35 & $\mathrm{~A} 2$ & zidovudina/lamivudina/efavirenz & 0,88 & $9,5 / 4,1$ & 8,3 & Normal \\
\hline 6 & 60 & $\mathrm{~A} 2$ & $\begin{array}{l}\text { zidovudina/lamivudina/nelfinavir } \rightarrow \text { tenofovir/abacavir/ } \\
\text { efavirenz }\end{array}$ & 1,13 & $9,3 / 2,2$ & 7,5 & Osteopenia cadera derecha \\
\hline 7 & 35 & A3 & tenofovir/emtricitabina/efavirenz & 1,15 & $9,7 / 2,3$ & 15,4 & Normal \\
\hline 8 & 46 & B3 & $\begin{array}{l}\text { zidovudina/lamivudina/efavirenz } \rightarrow \text { abacavir/lamivudina/ } \\
\text { efavirenz }\end{array}$ & 0,91 & $9,7 / 2,5$ & 8,2 & Osteopenia cadera izquierda \\
\hline 9 & 50 & $\mathrm{~A} 2$ & tenofovir/emtricitabina/efavirenz & 1,04 & $9,2 / 2,6$ & 28,4 & Osteopenia lumbar y caderas \\
\hline 10 & 46 & $\mathrm{~A} 2$ & abacavir/lamivudina/efavirenz & 1,06 & $10,2 / 2,7$ & 8,3 & Normal \\
\hline 11 & 47 & $\mathrm{~A} 2$ & tenofovir/emtricitabina/efavirenz & 0,8 & $9,7 / 3,2$ & 11,1 & Osteopenia lumbar \\
\hline 12 & 40 & $A 2$ & zidovudina/lamivudina/efavirenz & 0,89 & $10 / 3,3$ & 12,5 & Normal \\
\hline 13 & 60 & $\mathrm{~A} 2$ & $\begin{array}{l}\text { zidovudina/lamivudina/efavirenz } \rightarrow \text { abacavir/lamivudina/ } \\
\text { efavirenz }\end{array}$ & ND & $9,3 / 3,6$ & 6,3 & Osteopenia caderas \\
\hline 14 & 29 & $A 2$ & tenofovir/emtricitabina/efavirenz & 0,74 & $9,1 / 2,79$ & 6,3 & Osteopenia lumbar \\
\hline 15 & 46 & $A 2$ & abacavir/lamivudina/ atazanavir/ritonavir & 1,14 & $10,1 / 3,3$ & 20,9 & $\begin{array}{l}\text { Osteoporosis lumbar y } \\
\text { osteopenia caderas }\end{array}$ \\
\hline 16 & 46 & $\mathrm{~A} 3$ & zidovudina/lamivudina/nevirapina & 1,24 & $9,6 / 2,7$ & 19,2 & Normal \\
\hline
\end{tabular}


alcohol. Ocho de los 10 pacientes estaban en etapa A del CDC al momento del diagnóstico. Todos tenían carga viral menor a 50 copias $/ \mathrm{ml}$ y linfocitos T CD4 mayor a 350 céls $/ \mathrm{mm}^{3}$.

Seis pacientes usaban tenofovir y/o inhibidores de proteasa. Todos presentaban niveles bajos de $25 \mathrm{OHD}$ ( $50 \%$ con niveles deficientes), dos tenían niveles bajos de fósforo sérico (uno de ellos usuario de tenofovir) y dos tenían altos niveles de fosfatasas alcalinas. Todos presentaban niveles normales de calcio sérico.

\section{Discusión}

La incidencia de osteopenia u osteoporosis es mayor en individuos con infección por VIH que en la población general. A la fecha, no se han reportado datos sobre densidad mineral ósea en Chile o en Latinoamérica en este grupo de pacientes.

En esta muestra de 16 pacientes atendidos en un centro universitario de Santiago de Chile, observamos una muy alta frecuencia de baja densidad mineral ósea (63\% con osteopenia u osteoporosis) y la mayoría de los pacientes tenían niveles bajos de vitamina D. Ninguno de estos pacientes presentaban factores de riesgo mayores para osteoporosis ${ }^{11}$ como, edad $\geq$ a 65 años, historia de fracturas, terapia con glucocorticoides sistémicos o síndrome de malabsorción. Sin embargo, más de la mitad eran fumadores, un factor de riesgo menor para esta condición ${ }^{11}$. Cabe destacar que $87 \%$ de los pacientes del estudio fue estudiado en invierno, lo que podría haber aumentado la frecuencia de hipovitaminosis D.

La alta frecuencia de baja densidad mineral ósea y de hipovitaminosis D en este pequeño grupo de pacientes es una situación preocupante, ya que esto podría reflejar una condición prevalente en un mayor número de pacientes en nuestra región.

Próximamente iniciaremos un estudio prospectivo, con un mayor número de pacientes y con un seguimiento a 96 semanas, con el objetivo de determinar la prevalencia de este problema en nuestra población de pacientes con infección por VIH.

\section{Resumen}

Objetivo: El objetivo de este estudio fue determinar la frecuencia de hipovitaminosis D y de baja densidad mineral ósea en una muestra de pacientes con infección por VIH atendidos en una red de salud universitaria. Métodos: Una muestra aleatoria de 16 pacientes con infección por VIH fue seleccionada para la medición de niveles plasmáticos de vitamina D y densidad mineral ósea por densitometría. Resultados: 94\% de los pacientes mostraron bajos niveles de vitamina D. De ellos, 8 presentaron niveles insuficientes $(10-30 \mu \mathrm{g} / \mathrm{ml})$ y 7 , niveles deficientes (menor a $10 \mu \mathrm{g} / \mathrm{ml}$ ) de esta vitamina. Diez pacientes del total $(63 \%)$ presentaron una densitometría ósea anormal (osteopenia u osteoporosis). Todos los pacientes con osteopenia u osteoporosis tenían bajos niveles de vitamina $\mathrm{D}$, estaban en etapa A del CDC, presentaban recuento de linfocitos CD4 mayor a 350 céls/mm3 y carga viral de VIH menor a 50 copias $/ \mathrm{ml}$. Conclusión: En esta muestra de pacientes con infección por VIH atendidos en una red de salud universitaria de Chile existe una alta frecuencia de hipovitaminosis D y baja densidad mineral ósea.

\section{Referencias bibliográficas}

1.- Welz T, Childs K, Ibrahim F, Poulton M, Taylor C B, Moniz C F, et al. Efavirenz is associated with severe vitamin D deficiency and increased alkaline phosphatase. AIDS 2010; 24: $1923-8$

2.- Cozzolino M, Vidal M, Arcidiacono M V, Tebas P, Yarasheski K E, Dusso A S. HIV-protease inhibitors impair vitamin D bioactivation to 1,25-dihydroxyvitamin $\mathrm{D}$. AIDS 2003; 17: 513-20.

3.- Calmy A, Fux C A, Norris R, Vallier N, Delhumeau C, Samaras K, et al. Low bone mineral density, renal dysfunction, and fracture risk in HIV infection: a cross-sectional study. J Infect Dis 2009; 200: 1746-54.

4.- Womack J A, Goulet J L, Gibert C, Brandt C, Chang C C, Gulanski B, et al. Increased risk of fragility fractures among HIV infected compared to uninfected male veterans. PLoS One 2011; 6: e17217.

5.- Triant V A, Brown T T, Lee H, Grinspoon S K. Fracture prevalence among human immunodeficiency virus (HIV)-infected versus non-HIV-infected patients in a large U.S. healthcare system. J Clin Endocrinol Metab 2008; 93: 3499-504.

6.- Grund B, Peng G, Gibert C L, Hoy J F, Isaksson R L, Shlay J C, et al. Continuous antiretroviral therapy decreases bone mineral density. AIDS 2009; 23: 1519-29.

7.- Duvivier C, Kolta S, Assoumou L, Ghosn J, Rozenberg S, Murphy R L, et al. Greater decrease in bone mineral density with protease inhibitor regimens compared with nonnucleoside reverse transcriptase inhibitor regimens in HIV-1 infected naive patients.
AIDS 2009; 23: 817-24.

8.- Paccou J, Viget N, Legrout-Gérot I, Yazdanpanah Y, Cortet B. Bone loss in patients with HIV infection. Joint Bone Spine 2009; 76: $637-41$.

9.- McComsey G A, Tebas P, Shane E, Yin M T, Overton E T, Huang J S, et al. Bone disease in HIV infection: a practical review and recommendations for HIV care providers. Clin Infect Dis 2010; 51: 937-94.

10.- González G, Arriagada M, Jervis R. La función de la vitamina $\mathrm{D}$ en el manejo de la osteoporosis: Consenso Latinoamericano. Rev Chil Endocrinol Diabetes 2010; 3 (4): 293-8.

11.- Brown J P, Josse R G. Clinical practice guidelines for the diagnosis and management $\mathrm{f}$ osteoporosis in Canada. CMAJ 2002; 167 (10 Suppl): S1-S34. 